\title{
Clinical features of dopamine agonist withdrawal syndrome in a movement disorders clinic
}

\author{
Margarita Pondal, Connie Marras, Janis Miyasaki, Elena Moro, \\ Melissa J Armstrong, Antonio P Strafella, Binit B Shah, Susan Fox, \\ L K Prashanth, Nicolas Phielipp, Anthony E Lang
}

The Morton and Gloria Shulman Movement Disorders Centre and the Edmond J. Safra Program in Parkinson's Disease, University Health Network and University of Toronto, Toronto, Ontario, Canada

\section{Correspondence to} Dr Margarita Pondal, Movement Disorders Centre, Toronto Western Hospital, University of Toronto, and the Edmond J. Safra Program in Parkinson's Disease, McLaughlin Pavilion, 7th Floor, 399 Bathurst Street, Toronto, ON M5T 2S8, Canada; mpondals@gmail.com

Received 15 March 2012 Revised 12 July 2012

Accepted 1 August 2012

Published Online First

29 August 2012

\section{SLinked}

- http://dx.doi.org/10.1136/ jnnp-2012-303570

\section{ABSTRACT}

Background Recently, symptoms similar to addictive drug withdrawal have been reported in a structured longitudinal study of patients with idiopathic Parkinson's Disease (PD) withdrawing from dopamine agonists (DA): the dopamine agonist withdrawal syndrome (DAWS). Objectives The objective of this study was to establish the frequency, predictors, and outcomes of DAWS in a movement disorders clinic.

Methods We conducted a retrospective chart review of a sample of patients with a clinical diagnosis of PD treated with DA in whom withdrawal or attempted withdrawal of DA was carried out because of adverse effects, or for any other reason. Out of 487 PD patient charts reviewed, 84 were withdrawn from the agonists and were evaluable.

Results Thirteen patients (15.5\%) met criteria for DAWS (DAWS +) and 71 did not (DAWS-). DAWS developed upon withdrawal from pergolide, pramipexole and ropinirole, and did not respond to levodopa. DAWS outcomes included recovery in less than 6 months in $61 \%$, in more than a year in $23 \%$, and an inability to discontinue DA in $15 \%$ of patients. Development of impulse control disorders was the reason for DA withdrawal in all DAWS +, but only in $41 \%$ of DAWS patients $(p<0.0001)$. DAWS + and DAWS - patients did not differ in other variables.

Conclusion DAWS is a disabling complication of DA use. Critical features of the syndrome are the strong link with impulse control disorders, possibly the independence of DA dosage and type, and the resistance to treatment, including levodopa. Further studies are required to characterise those at risk as well as to define an effective treatment.

\section{INTRODUCTION}

Dopamine is a critical neurotransmitter in mesocorticolimbic circuits involved in reward. ${ }^{1-3}$ Drugs that stimulate this circuit, such as amphetamines and cocaine, are a major societal cause of addiction. $^{4-6}$ Dopamine replacement therapies (DRT) replenish the nigrostriatal pathway to control the motor symptoms of Parkinson's disease (PD), but also stimulate mesocorticolimbic circuits that are relatively intact, ${ }^{2-5}$ resulting in several addiction-related syndromes. ${ }^{4} 7$

Among substance addiction syndromes reported in patients with PD on DRT, the dopamine dysregulation syndrome (DDS) is characterised by compulsive drug consumption accompanied by psychomotor agitation and euphoria, drug-related dyskinesias, resistance to dose reduction and withdrawal symptoms characterised by depression, anxiety and impairment in occupational and social functioning. 389

Impulse control disorders (ICD), commonly involving pathological gambling, hypersexuality, compulsive buying and compulsive eating, constitute a second type of DRT-related disinhibitory psychopathology considered a behavioural addition. ${ }^{10}$ ICD appear much more commonly in patients treated with dopamine agonists (DA), in whom they are reported in $14-17 \%$ of cases, while in patients with levodopa treatment the frequency ranges from $0.7 \%$ to $7 \%$. ${ }^{4} 610$

The clinical manifestations of dopamine agonist withdrawal syndrome (DAWS) resemble other psychostimulant withdrawal syndromes and the observed lack of response to levodopa, antidepressants and anxiolytics and the improvement with DA replacement are consistent with a drug-specific withdrawal syndrome. ${ }^{7}$ Rabinak and Nirenberg ${ }^{7}$ postulated that the patients with DAWS belong to a 'mesocorticolimbic variant' of PD, with disproportionate mesocorticolimbic versus nigrostriatal dopaminergic dysfunction, increased vulnerability to DAWS and ICD.

Most patients with ICD do not use DRT in a compulsive manner. ${ }^{11-14}$ On the other hand, the dose escalation in DDS patients can be accompanied by the development of ICD. ${ }^{4}$ Okai et al ${ }^{15}$ reviewed the problems of diagnosing and assessing the severity of ICD and proposed an extension to the criteria for DDS diagnosis.

The apathy that develops in $\mathrm{PD}$ patients following subthalamic nucleus stimulation has been linked to the drastic reduction in medication after surgery, and can be considered a DRT-related withdrawal syndrome. ${ }^{16}$ In contrast to this restricted postsurgical withdrawal syndrome, in a cohort of PD patients enrolled in a structured longitudinal study, Rabinak and Nirenberg ${ }^{7}$ reported symptoms similar to addictive drug withdrawal developing on withdrawal or attempted withdrawal of DA. They defined the DAWS as a severe stereotyped cluster of physical and psychological symptoms that correlate with DA withdrawal in a dose-dependent manner, cause clinically significant distress or social/occupational dysfunction, are refractory to levodopa and other 
PD medications, and cannot be attributed to other clinical factors. ${ }^{7}$ DAWS comprises psychiatric symptoms such as anxiety, panic attacks, depression, agitation, irritability, dysphoria, insomnia, fatigue, generalised pain, and drug cravings and autonomic signs and symptoms such as orthostatic hypotension, dizziness, nausea and diaphoresis. ${ }^{7}$

We aimed to determine the frequency of DAWS, the determinants for developing this syndrome, and the outcome of DAWS in a population of PD patients followed in a movement disorders clinic

\section{METHODS}

We carried out a retrospective chart review of $\mathrm{PD}$ patients attending a large movement disorders clinic at the Toronto Western Hospital. The study project was approved by the Research Ethics Board of University Health Network, Toronto, Ontario, Canada.

Patient selection was carried out searching our database for a diagnosis of PD among patients with ongoing follow-up. All the information present in the chart was evaluated by a neurologist assessor. If clarification was required, and in all patients in whom DAWS was suspected, the case was discussed in detail with the attending neurologist. The inclusion criteria were a clinical diagnosis of PD using UK Parkinson's Disease Society brain bank criteria ${ }^{17}$ with a history of DA treatment for more than 3 months, in whom withdrawal of a DA had been carried out. Exclusion criteria were dementia as a clinical diagnosis stated in the chart, a history of functional neurosurgery for treatment of PD temporally close to withdrawal and insufficient information on DA treatment or withdrawal.

All data collection was completed before rendering a diagnosis of DAWS, which was retrospectively applied, upon reviewing the clinical information and the opinion of the patient's neurologist, using the criteria proposed by Rabinak and Nirenberg $^{7}$ : a severe stereotyped cluster of physical and psychological symptoms that correlated with DA withdrawal in a dosedependent manner, caused clinically significant distress or social/ occupational dysfunction, were refractory to levodopa and other PD medications (apart from DA), and could not be attributed to other clinical factors. Psychiatric symptoms included anxiety, panic attacks, depression, agitation, irritability, dysphoria, insomnia, fatigue, generalised pain, and drug cravings and autonomic signs and symptoms included orthostatic hypotension, dizziness, nausea and diaphoresis. ${ }^{7}$ The symptoms did not improve with levodopa, even in the 'on' state, but did improve with DA repletion. Finally, the symptoms could not be explained by other clinical factors. ${ }^{7}$

According to the chart description of functional impairment by the patients themselves, their family and caregivers, and patients' follow-up, the severity of DAWS was classified by the assessor as either mild-moderate if impairment was limited to demanding activities or if it had some impact on the patient's and family daily life, or severe if there was considerable disruption of the patient's and family daily life or inability to perform basic activities of daily life. The duration of DAWS symptoms was classified as remitting and lasting less than 6 months, 6 months to 1 year, or over a year, or not remitting and unable to withdraw from DA.

In addition to the gender and marital status, the following clinical variables were recorded: age at DA withdrawal, age at PD onset, $\mathrm{PD}$ duration at withdrawal time, history of smoking, and smoking at DA withdrawal time, ICD before PD, illicit drug use before PD. On the state unified Parkinson's disease rating scale (UPDRS) motor subscale scores were recorded during routine visits at DA withdrawal time. The levodopa equivalent daily dose (LEDD) was calculated as: regular levodopa plus 0.75 times the dose of continuous release levodopa plus 1.3 times the dose of levodopa/entacapone plus 100 times the dose of pramipexole dihydrocloride or pergolide mesylate plus 20 times the dose of ropinirole hydrochloride. ${ }^{7} 18$ Treatment variables recorded were: reason for DA withdrawal, specific DA withdrawn, dosage of levodopa (LD-LEDD), dosage of DA (DA-LEDD), total LEDD (DA-LEDD plus LD-LEDD) at withdrawal, cumulative dose exposure for DA (maintenance DA-LEDD times years of treatment), levodopa treatment duration and DA treatment duration in years.

The ICD diagnosis was clinical, rendered by the patient's neurologist, and registered in the chart when appropriate symptoms were reported by patients, their families and caregivers. In patients with ICD as the cause of withdrawal, the speed of DA withdrawal (DA-LEDD decrease per week), previous ICD with other DA, type of ICD, and duration of ICD before withdrawal were recorded. Patients with a diagnosis of DAWS (DAWS +) were compared with patients without (DAWS-); this was done for all patients, and a second comparison was restricted to patients withdrawn from DA due to ICD.

Categorical variables were compared using the $\chi^{2}$ test if the number expected in each cell was five or greater, and utilising Fisher's exact test otherwise. Continuous variables with a normal distribution as determined by the Kolmogorov-Smirnov test were compared by Student's t test, and otherwise by the nonparametric Mann-Whitney U test. No correction for multiple comparisons was applied. Statistical analysis was carried out using SPSS V.13.0 for Windows statistical software. Significance was set at $\mathrm{p}<0.05$.

\section{RESULTS}

Four hundred and eighty-seven active charts were reviewed; 297 patients (61\%) had been treated with a DA, and in 98 (33\%) of these DA were discontinued. Fourteen of these 98 patients were excluded from analysis, 10 because of insufficient data on treatment or withdrawal follow-up, two because they underwent PD surgery close to the time of DA withdrawal, and two because they had dementia at the time of DA withdrawal (figure 1)

Five patients had a remote history of functional PD surgery before withdrawal; none of them was withdrawn from DA because of ICD. ICD were the most common reason for DA withdrawal, representing $50 \%$ of the withdrawals $(n=42)$. Other

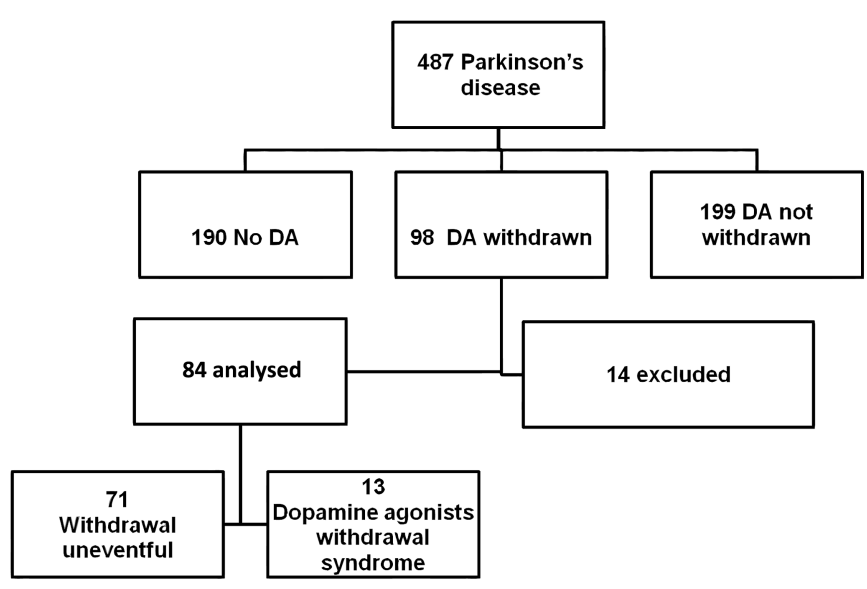

Figure 1 Patient flow chart. DA, dopamine agonist. 
causes were hypersomnia (thirteen), hallucinations (seven), worsening PD symptoms (five), nausea (five), cognitive impairment (five), hypotension (four), limb oedema (two) and isolated anxiety (one).

Out of 84 patients analysed, 13 (15.5\%) fulfilled the diagnostic criteria for DAWS, and 71 patients did not develop DAWS on withdrawal from DA (figure 1).

ICD were the reason for DA withdrawal in all 13 DAWS+ patients $(100 \%)$, compared to an ICD frequency of $41 \%$ in DAWS - patients (29 of 71), $(p<0.0001)$.

Each patient with DAWS had at least four (mean 4.9, median 5) of the 13 clinical manifestations suggestive of DAWS observed. These symptoms represented a change from their previous functioning level and were related in time to DA discontinuation. The most common of these were depressed mood in eleven patients (84\%), fatigue in ten (77\%), anxiety in nine $(69 \%)$ and insomnia in eight (61\%). Autonomic dysfunction was recorded in six patients $(46 \%)$ including diaphoresis, dizziness, nausea and flushed face; four patients (31\%) had panic attacks, four (31\%) generalised pain, three (23\%) irritability, three $(23 \%)$ drug cravings and one loss of interest $(8 \%)$. One patient voiced suicidal thoughts during the withdrawal period.

None of the clinical or treatment variables evaluated (see Methods section) significantly differed between DAWS + and DAWS - patients (table 1).

DAWS was observed in subjects withdrawn from pergolide (one), pramipexole (five) and ropinirole (seven); there was no statistically significant difference in the frequency of DAWS between the specific DA. In the group of patients withdrawn from DA because of ICD there was a trend towards a more frequent use of ropinirole than other DA (Fisher's exact test 0.043).

The levodopa dose was increased in all patients at the time of withdrawal. Three of the DAWS + patients increased levodopa dosages by $15-20 \%$ above the dose recommended by their physician in an attempt to treat DAWS symptoms after DA withdrawal: one of them had a history of DDS years before DA withdrawal and another developed DDS approximately 1 year after recovering from DAWS. Among the DAWS - patients who were withdrawn from DA because of ICD, just one had a history of DDS. DAWS symptoms were not abated in any patient by increasing the dose of levodopa. The degree of functional impairment was classified as mild-moderate in seven patients and severe in six (table 2).

There was no evident relationship between either the degree of functional impairment or the evolution of DAWS and the LDLEDD, DA-LEDD, cumulative DA-LEDD or total LEDD at withdrawal. Regarding the evolution of DAWS, eight patients recovered in less than 6 months, no patient had a resolution of symptoms between 6 months and 12 months, recovery took over a year in three patients, and two patients were unable to remain off of DA due to disabling symptoms of DAWS. These patients had persistent, chronic ICD associated with an inability to wean the DA (table 2).

Forty-two patients underwent DA withdrawal because of ICD, 13 DAWS + and 29 DAWS -. Table 3 shows the frequencies of ICD in DAWS+ and DAWS- patients. There were no statistical differences in the type of ICD between the DAWS+ and DAWS - groups (table 3).

Among the multiple demographic, clinical and treatment variables compared, the only differences within the group of patients withdrawn from DA because of ICD were that DAWS + patients were taking higher doses of levodopa (expressed in LEDD) at the time of DA withdrawal $(p<0.02)$ and had a more frequent smoking history $(p<0.03)$ (table 4$)$.

\section{DISCUSSION}

Our retrospective chart review in a clinic practice setting confirms the concept of DAWS as identified by Rabinak and Nirenberg. ${ }^{7}$ Using the same diagnostic criteria, we found a very similar frequency of the syndrome among those patients in whom DA were withdrawn, $15.5 \%$ in our series versus $19 \%$ in theirs. A frequency of $7.8 \%$ of patients withdrawn from DA was

Table 1 Clinical and treatment variables: comparison between DAWS + and DAWS - patients

\begin{tabular}{|c|c|c|c|}
\hline Clinical variables & DAWS $+(n=13)$ & DAWS $-(n=71)$ & $\mathrm{p}$ Value \\
\hline Age at PD onset & $51.8(7.2)(42-67)$ & $52.6(10.9)(28-79)$ & $0.73 \dagger$ \\
\hline PD duration at withdrawal & $9.1(5.4)(3-19)$ & $10(6.2)(1-30)$ & $0.72 \ddagger$ \\
\hline Age at withdrawal & $60.7(6.7)(46-70)$ & $62.7(11.2)(37-92)$ & $0.55 \dagger$ \\
\hline Gender ( $\%$ male) & $69 \%$ & $75 \%$ & $0.68^{*}$ \\
\hline Marital status (\% married) & $82 \%$ & $81 \%$ & $0.95^{*}$ \\
\hline UPDRS-III score at withdrawal & $19(5.3)(8-27)$ & $23.7(10.7)(6-50)$ & $0.18 \neq$ \\
\hline Smoking history (\%) & $45 \%$ & $22 \%$ & $0.10^{*}$ \\
\hline Smoking at withdrawal & $7.6 \%$ & $6 \%$ & $0.7^{*}$ \\
\hline ICD before PD (\%) & $8 \%$ & $3 \%$ & $0.4^{*}$ \\
\hline Illicit drug use before PD (\%) & $8 \%$ & $3 \%$ & $0.4^{*}$ \\
\hline Treatment variables & DAWS + & DAWS- & $\mathrm{p}$ Value \\
\hline LD-LEDD at DA & $842(470)(300-1800)$ & $625(540)(0-2000)$ & $0.11 \neq$ \\
\hline Levodopa years of treatment at DA withdrawal & $5.3(4.5)(1-13)$ & $7(6.4)(0-26)$ & $0.7 \neq$ \\
\hline DA-LEDD at DA withdrawal & $239(142)(60-480)$ & $215(145)(40-600)$ & $0.47 \neq$ \\
\hline Total LEDD at withdrawal & $1051(507)(300-1900)$ & $841(563)(40-2450)$ & $0.13 \neq$ \\
\hline Cumulative DA-LEDD (dose $\times$ years) & $1335(1124)(15-3525)$ & $949(1136)(15-4612)$ & $0.08 \neq$ \\
\hline DA treatment duration (years) & $4.9(3.3)(0.25-12)$ & $3.9(3.5)(0.25-17)$ & $0.15 \neq$ \\
\hline
\end{tabular}


Table 2 Degree of functional impairment and outcome in DAWS+

\begin{tabular}{|c|c|c|c|c|}
\hline \multicolumn{5}{|c|}{ Functional impairment of DAWS + patients } \\
\hline \multicolumn{5}{|c|}{ Mild to moderate: limited to demanding activities or if it had some impact on the patient's and family daily life } \\
\hline $\mathrm{n}$ & LD-LEDD (mg) p p & DA-LEDD (mg) $\mathrm{p}^{2}$ & Cumulative DA-LEDD (mg) $\mathrm{p}^{3}$ & Total LEDD at withdrawal $p^{4}$ \\
\hline 7 & $963(562)(300-1800)$ & $226(167)(60-480)$ & $1445(1453)(15-3525)$ & $1189(558)(420-1900)$ \\
\hline \multicolumn{5}{|c|}{ Severe: considerable disruption of the patient's and family daily life or inability to perform basic activities of daily life } \\
\hline $\mathrm{n}$ & LD-LEDD (mg) & DA-LEDD (mg) & Cumulative DA-LEDD (mg) & Total LEDD at withdrawal \\
\hline 6 & $702(328)(300-1200)$ & $255(120)(120-450)$ & $1208(679)(600-2475)$ & $957(306)(560-1320)$ \\
\hline \multicolumn{5}{|c|}{ Outcome of DAWS patients } \\
\hline \multicolumn{5}{|c|}{ Recovery $<6$ months } \\
\hline $\mathrm{n}$ & LD-LEDD (mg) p ${ }^{5}$ & DA-LEDD (mg) $p^{6}$ & Cumulative DA-LEDD (mg) $\mathrm{p}^{7}$ & Total LEDD at withdrawal $p^{8}$ \\
\hline 8 & $930(540)(300-1800)$ & $213(126)(60-400)$ & $1274(1391)(15-3525)$ & 1143 (549) (420-1900) \\
\hline \multicolumn{5}{|c|}{ Recovery $>6$ months $<1$ year } \\
\hline 0 & - & - & - & - \\
\hline \multicolumn{5}{|c|}{ Recovery $>1$ year } \\
\hline 3 & 750 (409) (400-1200) & $350(199)(120-480)$ & $1710(662)(1325-2475)$ & $1100(220)(880-1320)$ \\
\hline \multicolumn{5}{|c|}{ Unable to tolerate DA withdrawal due to DAWS symptoms } \\
\hline 2 & $633(329)(400-866)$ & $180(28)(160-200)$ & 1019 (299) (807-1231) & $813(357)(560-1066)$ \\
\hline
\end{tabular}

reported in the only other study that we are aware of that examined DAWS. ${ }^{19}$ Unlike our study and that of Rabinak and Nirenberg, ${ }^{7}$ that series included subjects who had undergone deep brain stimulation (DBS) surgery while DA were discontinued, and thus some confusion with the postsurgical apathyrelated features (see below) may have been possible.

Similarities in the results of our study and that of Rabinak and Nirenberg ${ }^{7}$ include the frequency of ICD as the cause of withdrawal from DA (50\% vs $58 \%$ ), the proportion of subjects who developed DAWS when ICD were the reason for DA withdrawal (approximately one third in both series), and the 100\% association between DAWS and ICD, contrasting with the $34 \%$ reported by Limotai et al. ${ }^{19}$ Previous ICD were significantly more common in DAWS + than in DAWS - cases $(p<0.0001)$. Compared to the magnitude of this effect, all other variables studied were either without effect or at most of marginal significance.

In our study the patients who attempted to improve their withdrawal symptoms by increasing their levodopa dose by 15-20\% more than prescribed by their physician did not have other clinical manifestations suggestive of DDS at this time. DAWS symptoms did not disappear with equivalent or higher doses of dopaminergic medications other than DA, as previously reported. ${ }^{7}$ We cannot exclude the possibility that there may be a milder group of 'treatable DAWS' patients who had subtle

Table 3 Addictive behaviours in patients withdrawing because of ICD

\begin{tabular}{lllc}
\hline Clinical variables & $\begin{array}{l}\text { DAWS+ } \\
(\mathbf{n = 1 3})\end{array}$ & $\begin{array}{l}\text { DAWS } \\
(\mathbf{n = 2 9 )}\end{array}$ & p Value* \\
\hline Compulsive sexual behaviour & $6(46 \%)$ & $11(38 \%)$ & 0.61 \\
Compulsive gambling & $6(46 \%)$ & $19(66 \%)$ & 0.23 \\
Compulsive eating & $3(23 \%)$ & $7(24 \%)$ & 0.94 \\
Compulsive shopping & $2(15 \%)$ & $2(6.9 \%)$ & 0.38 \\
Compulsive computer behaviour & $1(8 \%)$ & $0(0 \%)$ & - \\
More than one ICD & $4(30 \%)$ & $8(28 \%)$ & 0.83 \\
Punding & 0 & $1(3 \%)$ & \\
\hline Results are given as size and percentage. & & \\
${ }^{*} \chi^{2}$ test. & & & \\
DAWW, dopamine agonist withdrawal syndrome; ICD, impulse control disorder.
\end{tabular}

DAWS symptoms and who responded to levodopa adjustment. Mild self-limited withdrawal symptoms not meeting the DAWS criteria of Rabinak and Nirenberg ${ }^{7}$ that we used in our study were included in a recent report of patients tapering DA. ${ }^{20}$ These patients would have been diagnosed as DAWS - in our study and it is not at all clear that the reported milder symptoms of low mood or apathy have the same pathogenic basis as the more complex psychostimulant drug withdrawal-like symptoms of DAWS. Self-medication with supratherapeutic doses of levodopa in an attempt to alleviate DAWS symptoms ${ }^{7}$ and the significant association reported in one series between DAWS and DDS ${ }^{19}$ suggest an overlapping vulnerability to DDS in the DAWS+ patients. This tendency of DAWS + patients to escalate levodopa dosage is also consistent with 'negative reinforcement' models of addiction. ${ }^{2}$ Okai et al ${ }^{15}$ considered that patients with DDS and even non-DDS patients often take extra medication to avoid the distress and dysphoria that appears during the off state, and propose that this pattern is similar to that of DAWS. In our opinion the two entities differ in that DAWS is related to a drug withdrawal situation, in contrast to the steady or escalating dose of levodopa in DDS patients, and the spectrum of symptoms of DAWS is broader and more typical of an addictive drug withdrawal. The symptoms of DAWS were extremely disruptive to the patients and their families: in almost $40 \%$ of patients, DAWS symptoms were either associated with a protracted course, taking over a year to withdraw the offending agent fully, or they were refractory to attempts to withdraw DA; these patients required ongoing treatment with the result that their ICD continued unabated.

The average age of PD onset in our series of patients is just over 50 years and below that expected in the general population. ${ }^{21}$ The presence of dementia as a clinical diagnosis stated in the chart as one of the exclusion criteria could have contributed to reducing the number of older patients included in our series. Our study was not designed to determine the frequency of ICD in PD patients treated with DA. Pathological gambling was the type of ICD most commonly observed, as reported by Voon et al. ${ }^{13}$ The frequency of multiple ICD, observed in $28 \%$ of all our ICD patients, is similar to the one quarter ${ }^{6}$ and one third ${ }^{22}$ reported in other surveys. On the other hand, in patients with DAWS Rabinak and Nirenberg ${ }^{7}$ observed a much higher $(80 \%)$ 
Table 4 Clinical and treatment variables: patients withdrawn from DA due to ICD $(n=42)$

\begin{tabular}{|c|c|c|c|}
\hline & DAWS $+(n=13)$ & DAWS $-(n=29)$ & p Value \\
\hline \multicolumn{4}{|l|}{ Clinical variables } \\
\hline Age at PD onset & $51.8(7.2)(42-67)$ & $49.9(9.5)(30-69)$ & $0.47 \dagger$ \\
\hline PD duration at withdrawal (years) & $9.1(5.5)(3-19)$ & $9.4(5.7)(1-21)$ & $0.91 \neq$ \\
\hline Age at withdrawal & $60.7(6.7)(46-70)$ & $59.3(10.5)(42-84)$ & $0.65 \dagger$ \\
\hline Gender ( $\%$ male) & $69 \%$ & $82 \%$ & $0.32^{*}$ \\
\hline UPDSR-III score at withdrawal & $19(5.3)(8-27)$ & $21(9.9)(7-47)$ & $0.65 \ddagger$ \\
\hline Marital status (\%) & $82 \%$ & $82 \%$ & $0.98^{*}$ \\
\hline Smoking history (\%) & $45 \%$ & $14 \%$ & $0.03^{*}$ \\
\hline Smoking at withdrawal (\%) & $9 \%$ & $7 \%$ & $0.86^{*}$ \\
\hline ICD before PD & $8 \%$ & $7 \%$ & $0.68^{*}$ \\
\hline Illicit drug use before PD & $8 \%$ & $3 \%$ & $0.52^{*}$ \\
\hline $\begin{array}{l}\text { ICD duration before withdrawal } \\
\text { (months) }\end{array}$ & $12.3(13.1)$ & $12.6(14.1)$ & $0.9 \dagger$ \\
\hline ICD with previous DA (\%) & $17 \%$ & $57 \%$ & $0.09 *$ \\
\hline \multicolumn{4}{|l|}{ Treatment variables } \\
\hline LD-LEDD at DA withdrawal & $842(470)(300-1800)$ & $491(423)(0-1600)$ & $0.02 \ddagger$ \\
\hline $\begin{array}{l}\text { Levodopa treatment duration at } \\
\text { withdrawal }\end{array}$ & $5.3(4.5)(1-13)$ & $6.8(6.3)(0-21)$ & $0.88 \neq$ \\
\hline DA-LEDD at DA withdrawal & $239(142)(60-480)$ & $289(152)(40-600)$ & $0.37 \neq$ \\
\hline Total LEDD at withdrawal & $1051(507)(300-1900)$ & $780(503)(40-2100)$ & $0.11 \neq$ \\
\hline DA treatment duration (years) & $4.9(3.3)(0.25-12)$ & $4.7(4.1)(0.3-17)$ & $0.56 \neq$ \\
\hline Cumulative DA-LEDD (dose $\times$ years) & $1335(1124)(15-3525)$ & $1296(1217)(40-4404)$ & $0.78 \neq$ \\
\hline DA-LEDD withdrawn/week & $61(55)(9-200)$ & $53.4(54.3)(8-225)$ & $0.76 \ddagger$ \\
\hline \multicolumn{4}{|c|}{$\begin{array}{l}\text { Results are given as mean (SD), (range) unless otherwise indicate. } \\
{ }^{*} \chi^{2} \text { or Fisher's exact test. } \\
+\mathrm{t} \text { Test. } \\
\text { ‡Mann-Whitney U test. } \\
\text { Missing data: Marital status: DAWS+, 2; DAWS-, 1. UPDRS-III: DAWS }+, 3 \text {; DAWS-, 3. Smoking history: DAWS+, 2, DAWS-, 1. Smoking at withdrawal: DAWS+, 2; DAWS-, 2 } \\
\text { Speed of withdrawal (DA-LEDD/week): DAWS+, 1; DAWS-, 5; ICD duration at withdrawal: DAWS+, 1; DAWS-, 1. } \\
\text { DA, dopamine agonist; DAWS, dopamine agonist withdrawal syndrome; ICD, impulse control disorder; LEDD, levodopa equivalent daily dose; PD, Parkinson's disease; UPDRS, unified } \\
\text { Parkinson's disease rating scale. }\end{array}$} \\
\hline
\end{tabular}

frequency of multiple ICD than our 30\%. Among patients with ICD, there was no difference in the type or multiplicity of ICD between DAWS + and DAWS - in our study, suggesting that the presence of DA-induced ICD, rather than the type or complexity, is the relevant predictor for the development of DAWS.

A significantly lower UPDRS motor score (suggesting a less severe form or earlier PD stage) in DAWS + patients was reported previously, ${ }^{7}$ but we could not confirm this using onstate UPDRS scores obtained during routine clinic visits. Our failure to demonstrate a difference in motor symptomatology between DAWS + and DAWS - patients does not exclude a role of the mesocorticolimbic system in the pathogenesis of DAWS. However, we did not find evidence to support a disproportionate sparing of the motor circuit in these patients.

In contrast to other studies, we did not find either a higher baseline dose of DA in DAWS patients ${ }^{7}$ or higher DA cumulative exposure, $^{7} 19$ although a statistical trend towards higher cumulative DA-LEDD was observed $(p=0.08)$. When we restricted the comparison to the group with ICD, DAWS patients had a higher LD-LEDD at DA withdrawal $(p<0.02)$ than patients without DAWS. Similarly, the development of ICD is reported to be associated with higher LD-LEDD ${ }^{6}$; however, ICD can develop irrespective of DA dosage, ${ }^{6}$ and even with very low DA cumulative exposure in patients with restless legs syndrome. ${ }^{23-25}$

All DAWS + patients in the original report of Rabinak and Nirenberg ${ }^{7}$ were using pramipexole. We extend the concept of DAWS by describing this syndrome with other DA. We did not find statistically significant differences among the specific DA drugs in the frequency of development of DAWS in the total sample of 84 patients; however, the small numbers in each treatment group limit interpretation. Although in the group of patients withdrawn from DA because of ICD there was a trend towards a more frequent use of ropinirole than other DA (Fisher's exact test 0.043), we do not consider this result relevant because of the marginal significance. The RR of developing ICD with pramipexole compared to other DA has been disputed in the literature, but it is clear that several DA can induce the syndrome, and the general consensus is that this is a class effect. $^{6}{ }^{26-28}$ While reported cases of ICD in PD secondary to bromocriptine are scarce, ${ }^{29}$ the two patients withdrawn from bromocriptine in our series had ICD.

Among patients with ICD, a history of smoking (considered an addictive behaviour) was present more often in DAWS + than in DAWS - patients $(\mathrm{p}<0.03)$.

We did not correct for multiple comparisons. With this caveat it is interesting to note the trend we found in patients with ICD towards higher doses of dopamine replacement treatments and addictive behaviour in the DAWS + group compared to the DAWS - group. Our study has two major limitations. First, it is a retrospective chart review with all the acknowledged problems inherent with this approach. Second, it is purely clinical, and thus contributes no insights into the pathophysiological mechanisms or the genetic risk factors for the development of DAWS.

In conclusion, DAWS is an important clinical problem with manifestations resembling those of other drug withdrawal syndromes and a relevant concept in the management of patients with PD. The individual tendency to develop ICD in response to exposure to any DA, rather than other treatment or demographic factors, may be the major determinant in the development of DAWS. The fact that a smoking history in the 
ICD group increases the likelihood of developing DAWS suggests a susceptibility to addiction in these patients. A higher dose of levodopa may also contribute to the development of DAWS in patients with ICD.

Further prospective studies are required to determine the frequency and spectrum of severity of DAWS, and to define effective treatment for DAWS, given the potential for considerable disability and extended course experienced by many patients.

Contributors MP: contributed to the design of the study, reviewed charts, acquired data, introduced data in the database, analysed and interpreted data, drafted the first version of the article, modified the article introducing the authors' suggestions and approved the version to be published. CM: reviewed and discussed the patients' criteria for selection, participated in the analysis and interpretation of data, critically revised the manuscript for important intellectual content and approved the final version to be published. JM: preselected, reviewed and discussed the patients for selection criteria, critically revised the manuscript for important intellectual content and approved the final version to be published. EM, MJA, APS, BBS, SF, LKP and NP: reviewed and discussed the patients for selection criteria, critically revised the manuscript for important intellectual content and approved the final version to be published. AEL: originated the idea of the study, contributed to the design of the study, reviewed and discussed the patients for selection criteria, critically revised the manuscript for important intellectual content and approved the final version to be published.

Competing interests MP: received funds from University of Toronto for travelling and registering at the AAN meeting, Honolulu 2012, to present data related to the study that is being submitted. CM: consulting compensation from Solvay Pharmaceuticals. JM: Teva Consulting, clinical trial. National Institutes of Health. NET PD studies. Independent clinical monitor. Neurogen 2009 data safety monitor committee chair. EM: received honorarium from Medtronics. AS: governmental organisation grant. SF: scientific advisory board for Merck Serono, Merz. Merck Serono clinical trial, site PI. AL: consultant for research projects, drug development, etc. Abbott, Allon Therapeutics, Astra Zenica, Biovail, Boerhinger-Ingelheim, Cephalon, Ceregene, Eisai, Medtronic, Lundbeck A/S, Novartis, Merk Serono, Solvay, Teva. Expert witness: cases related to the welding industry. Canadian Institutes of Health Research, Dystonia Medical Research Foundation, Michael J. Fox Foundation, National Parkinson's Foundation, Ontario Problem Gambling Research Centre. MA, BS, LKP and NP: nothing to disclose.

Ethics approval Provided by the Research Ethics Board of University Health Network, Toronto, Ontario, Canada.

Provenance and peer review Not commissioned; externally peer reviewed.

\section{REFERENCES}

1. Evans AH, Strafella AP, Weintraub D, et al. Impulsive and compulsive behaviors in Parkinson's disease. Mov Disord 2009:24:1561-70.

2. Evans AH, Lawrence AD, Cresswell SA, et al. Compulsive use of dopaminergic drug therapy in Parkinson's disease: reward and anti-reward. Mov Disord 2010:25:867-76.

3. Giovannoni G, O'sullivan JD, Turner K, et al. Hedonistic homeostatic dysregulation in patients with Parkinson's disease on dopamine replacement therapies. J Neurol Neurosurg Psychiatry 2000;68:423-8.

4. Lim SY, Evans AH, Miyasaki JM. Impulse control and related disorders in Parkinson's disease: review. Ann NY Acad Sci 2008:1142:85-107.
5. Pezzella FR, Colosimo C, Vanacore N, et al. Prevalence and clinical features of hedonistic homeostatic dysregulation in Parkinson's disease. Mov Disord 2005;20:77-81.

6. Weintraub D, Koester J, Potenza MN, et al. Impulse control disorders in Parkinson disease: a cross-sectional study of 3090 patients. Arch Neurol 2010;67:589-95.

7. Rabinak CA, Nirenberg MJ. Dopamine agonist withdrawal syndrome in Parkinson disease. Arch Neurol 2010:67:58-63.

8. Evans $\mathbf{A H}$, Lees AJ. Dopamine dysregulation syndrome in Parkinson's disease. Curr Opin Neurol 2004;17:393-8

9. Lawrence AD, Evans AH, Lees AJ. Compulsive use of dopamine replacement therapy in Parkinson's disease: reward systems gone awry? Lancet Neurol 2003:2:595-604

10. O'Sullivan SS, Evans AH, Lees AJ. Dopamine dysregulation syndrome: an overview of its epidemiology, mechanisms and management. CNS Drugs 2009;23:157-70.

11. Nirenberg MJ. Dopamine agonist withdrawal syndrome and non-motor symptoms after Parkinson's disease surgery. Brain 2010:133:e155.

12. Gallagher DA, O'Sullivan SS, Evans AH, et al. Pathological gambling in Parkinson's disease: risk factors and differences from dopamine dysregulation. An analysis of published case series. Mov Disord 2007;22:1757-63.

13. Voon V, Hassan K, Zurowski M, et al. Prevalence of repetitive and reward-seeking behaviors in Parkinson disease. Neurology 2006:67:1254-7.

14. Voon V, Fernagut PO, Wickens J, et al. Chronic dopaminergic stimulation in Parkinson's disease: from dyskinesias to impulse control disorders. Lancet Neurol 2009;8:1140-9.

15. Okai D, Samuel M, Askey-Jones S, et al. Impulse control disorders and dopamine dysregulation in Parkinson's disease: a broader conceptual framework. Eur J Neurol 2011:18:1379-83.

16. Thobois S, Ardouin C, Lhommee E, et al. Non-motor dopamine withdrawal syndrome after surgery for Parkinson's disease: predictors and underlying mesolimbic denervation. Brain 2010;133:1111-27.

17. Hughes AJ, Daniel SE, Kilford L, et al. Accuracy of clinical diagnosis of idiopathic Parkinson's disease: a clinico-pathological study of 100 cases. J Neurol Neurosurg Psychiatry 1992;55:181-4.

18. Evans $\mathbf{A H}$, Katzenschlager R, Paviour D, et al. Punding in Parkinson's disease: its relation to the dopamine dysregulation syndrome. Mov Disord 2004:19:397-405.

19. Limotai N, Oyama G, Go C, et al. Addiction-like manifestations and Parkinson's disease: a large single center 9-year experience. Int J Neurosci 2012:122:145-53.

20. Cunnington AL, White L, Hood K. Identification of possible risk factors for the development of dopamine agonist withdrawal syndrome in Parkinson's disease. Parkinsonism Relat Disord. Published Online First: 6 June 2012

21. van Rooden SM, Heiser WJ, Kok JN, et al. The identification of Parkinson's disease subtypes using cluster analysis: a systematic review. Mov Disord 2010;25:969-78.

22. Weiss HD, Hirsch ES, Williams JR, et al. Detection of impulse control disorders in Parkinson disease patients. Neurologist 2010;16:406-7.

23. Tippmann-Peikert M, Park JG, Boeve BF, et al. Pathologic gambling in patients with restless legs syndrome treated with dopaminergic agonists. Neurology 2007;68:301-3.

24. d'Orsi G, Demaio V, Specchio LM. Pathological gambling plus hypersexuality in restless legs syndrome: a new case. Neurol Sci 2011;32:707-9.

25. Dang D, Cunnington D, Swieca J. The emergence of devastating impulse control disorders during dopamine agonist therapy of the restless legs syndrome. Clin Neuropharmacol 2011;34:66-70

26. Dodd ML, Klos KJ, Bower JH, et al. Pathological gambling caused by drugs used to treat Parkinson disease. Arch Neurol 2005:62:1377-81.

27. Szarfman A, Doraiswamy PM, Tonning JM, et al. Association between pathologic gambling and parkinsonian therapy as detected in the Food and Drug Administration adverse event database. Arch Neurol 2006:63:299-300.

28. Voon V, Hassan K, Zurowski M, et al. Prospective prevalence of pathologic gambling and medication association in Parkinson disease. Neurology 2006:66:1750-2.

29. Montastruc JL, Schmitt L, Bagheri H. Pathological gambling behavior in a patient withParkinson's disease treated with levodopa and bromocriptine. Rev Neurol (Paris) 2003;159:441-3. 